\title{
LA REPRESENTACIÓN DE LA MUJER EN LA ROMA DE LA POSGUERRA: EL TEATRO DE ENNIO FLAIANO
}

\author{
FEMALE REPRESENTATION IN POST-WAR ROME: THE THEATRICAL WORK OF \\ ENNIO FLAIANO
}

Milagro Martín Clavijo

Universidad de Salamanca

\section{Resumen:}

La mayoría de las mujeres que aparecen en la obra dramática del escritor italiano Ennio Flaiano (1910-1972) responden fundamentalmente al perfil general de mujer emancipada y liberada, quizás en exceso, al prototipo de mujer que aparece en La dolce vita de Fellini, en la que el autor colaboró como guionista. Son mujeres que no se presentan a sí mismas, sino a través de sus relaciones con los hombres protagonistas del teatro de Flaiano, de lo que les hacen sentir o no. La mayoría de ellas pertenecen al círculo intelectual-artístico de la Roma de la posguerra, mujeres liberadas pero que, en realidad, todavía desempeñan un papel secundario respecto al hombre. Mujeres en busca de la 'gran ocasión', el matrimonio, y es en este campo, el de las relaciones personales más íntimas en el que se van a mover todas las mujeres que aparecen en su teatro. Se trata fundamentalmente del amor, o más bien el deseo carnal, que parte de los personajes masculinos hacia las mujeres.

\section{Palabras claves:}

Ennio Flaviano, teatro, mujer, Roma.

\section{Abstract:}

Most of the women who appear in the plays of Italian writer Ennio Flaiano (1910-1972) represent the general profile of a emancipated and liberated woman, perhaps excessively, the prototype of the woman in Fellini's La dolce vita, film in which in which the author collaborated as a screenwriter. They are women who are not introduced by themselves, but through their relationships with the male main characters of the theater of Flaiano, from which men make them feel or not. Most of them belong to the intellectual-artistic society of post-war Rome, liberated women but who, actually, still play a supporting role regarding men. Women who are in search of the "great opportunity", marriage, the field of the most intimate relationships in which all the women of his theater appear. It is essentially about love, or rather the carnal desire, which comes from the male characters towards the female ones.

\section{KEY WORD:}

Ennio Flaiano, theater, woman, Rome. 


\section{El HOMbRe: PROtAgonista AbSOLUTO del teAtro de Flaiano}

EnlaproduccióndeFlaiano ${ }^{1}$ hay unclaropredominiodepersonajesmasculinos.Todos los protagonistasylamayor partedelos personajessecundariossonhombres. Las mujeres que aparecen en sus obras son muy pocas y están perfiladas con menos detalle que éstos. Parece como si a Flaiano le resultase más fácil expresar su visión de la vida, del mundo, a través de los hombres que son los que, presumiblemente, conoce mejor. Además, hay que matizar que sus personajes no solamente son hombres en su mayoría, sino que también prácticamente todos pertenecen a la esfera de los intelectuales, artistas y escritores por profesión, por vocación o por moda. Este es el mundo en el que el autor se desenvuelve con mayor facilidad, el que conoce y consigue expresar mejor. Queremos aclarar desde el primer momento que no consideramos que se trate de un personaje misógino. A nuestro parecer, su opción por el predominio de protagonistas masculinos no encierra ninguna trampa. Creemos que es a través del hombre -como varón- que el autor se refiere a los hombres, a la humanidad. Las situaciones que nos ofrece Flaiano protagonizadas por hombres no perderían nada si las protagonizasen mujeres, son situaciones personales, de crisis interior y profunda de la humanidad de nuestra época y que no tienen que ver con el sexo de la persona. Se trata del Hombre como representante de la especie humana. Hecha esta aclaración que nos parece fundamental, señalamos que, además, los personajes masculinos presentan una gran cantidad de rasgos en común con el escritor: hombres intelectuales, relacionados con la escritura y con el mundo del arte, en profunda crisis personal y, como escritores, rodeados de pseudointelectuales, de aristócratas y ricachones que ven en la inclinación hacia el arte un cierto toque 'chic' que está de moda y les puede sentar bien, dada la sociedad corrompida en la que se encuentran inmersos.

\section{EL PERSONAJE FEMENINO}

Las mujeres, como hemos visto, no desempeñan funciones decisivas en la sociedad que nos presenta Flaiano y tampoco las tendrán en su obra dramática. En

1 Ennio Flaiano (Pescara, 1910- Roma, 1972). Estudió arquitectura, pero se pasó enseguida al periodismo (Occidente, Oggi, Cine illustrato, Documento, Il Risorgimento liberale e Il Mondo) y a la crítica cinematográfica y teatral. Su actividad primordial es la de escritor, aunque es más conocido como crítico y guionista cinematográfico (durante los años cincuenta y sesenta Flaiano trabaja con Fellini en los guiones de I vitelloni, La dolce vita, 8 e $1 / 2$ y Giulietta degli spiriti, entre otros) que como dramaturgo. Flaiano publica obras de distintos géneros -poesía, novela, diario-, además de una gran abundancia de microtextos en forma de apuntes, aforismos, máximas y narraciones breves. Sus obras de teatro principales están incluidas bajo el título de Un marziano a Roma e altre farse (Flaiano 1971): La guerra spiegata ai poveri (1946), La donna nell'armadio (1957), Il caso Papaleo (1960), Un marziano a Roma (1960), La conversazione continuamente interrotta (1972). El año que figura entre paréntesis es el de su representación. Flaiano dejó muchas obras sin editar, e incluso algunas sin terminar, que se publicaron en un volumen conjunto. Tres de ellas son dramáticas: Come ogni giorno. Ballata romana, Dialogo per provare una penna nuova y Amleto '43. (Flaiano 1988). Todas las citas que aparecen en esta comunicación han sido tomadas de estas ediciones. este sentido, el papel de la mujer suele responder a arquetipos. La mayoría de las mujeres que aparecen en la obra dramática de Ennio Flaiano responden al perfil general de mujer emancipada y liberada, quizás en exceso, al prototipo de mujer que aparece en La dolce vita, de Fellini, en la que Flaiano colaboró como guionista. Son mujeres que pertenecen al círculo intelectual-artístico de la Roma de la posguerra. Mujeres liberadas pero que, en realidad, todavía desempeñan un papel secundario respecto al hombre. Mujeres en busca de la 'gran ocasión': el matrimonio.Como afirma Sergiacomo, las figuras femeninas son vistas y contadas siempre desde fuera, son objeto y no sujeto de representación:

"Ambite, sognate, ossessivamente desiderate e temute, ma in realtà come sempre inchiodate ad un ruolo edonistico e subalterno rispetto all'uomo, alcuni personaggi di donne emancipate si rivelano una riedizione in chiave satirica della donna di donne emancipate si rivelano una riedizione in chiave satirica della donna
fatale e vivono come protagoniste di una stupefacente collezione di storie zeppe di fatale e vivono come protagoniste di una stupefacente collezione di storie zeppe di
pruriti adulterini, gallismi, ninfomanie, di tradimenti presagiti e reciprocamente inflitti nell'implacabile gioco della coppia". (Sergiacomo 1993:116)

Esta es la visión más generalizada de la mujer en la obra de Flaiano, una visión nada positiva.Peronoconvienedejarseengañar:lasátiraqueelautorhacedelamujerformaparte delasátiradelasociedad ensutotalidad, noesunsectorquesatiricedemaneraprivilegiada. Las mujeres, por regla general, aparecen en el teatro de Flaiano unidas al tema del amor. Se trata de un amor -o un deseo carnal- que parte de los personajes-hombres hacia las mujeres. Éstas pueden ser reales o irreales, en el sentido de soñadas o recordadas, pero raramente están presentes. $\mathrm{Y}$ es hacia estas mujeres que los protagonistas van a experimentar un sentimiento contradictorio o, quizás, complementario: la maravilla y el tedio. Entre estos dos polos opuestos se encuentra tratado el tema de las mujeres en las obras dramáticas de Flaiano, aunque ya desde el principio se nos presenta de manera evidente qué tipo predomina.

\section{LA MUJER Y EL TEDIO}

La mujer y la relación amorosa que ella implica para el protagonista viene directamente relacionada con el tedio, con el vacío, con la superficialidad, con la nada en la que vive o malvive la sociedad de nuestra época. Respecto a este hastío que corroe al hombre moderno, Flaiano nos da las pautas en Il gioco e il massacro:

“La noia di Fabbro non viene dalla sazietà delle cose, ma dal sentimento della loro inutilità. Non prova nemmeno più emozioni morali: tutto è stato detto. Lavora senza ingannarsi, solo perché detesta il suicidio." (Flaiano 1988: 812) 
Esto es lo el autor entiende por tedio y nos lo hace llegar a través del comportamiento de sus personajes en su teatro. Se trata de un sentimiento de que todo es inútil, de que hagas lo que hagas nada tiene sentido. El hastío significa ser incapaces de sentir, de tener emociones por las personas, por las cosas. El hombre se ve reducido casi al estado vegetativo: se come, se duerme, se trabaja, se finge que se ama, que se lleva una vida como debeser, comola sociedad ha dictado que debellevarse una vida. En definitiva, se arrastra una vida hasta que seacabe. Quizás porquees mayorel miedo a perderla definitivamente. En Una e una notte nos encontramos también con personajes también aburridos que analizan este sentimiento de hastío:

"Provò a chiedersi di che cosa era scontento e dovette rispondersi che tutto andava abbastanza bene, ma che pure qualcosa lo amareggiava, una sensazione di noia, di disinteresse per i giorni che sarebbero venuti,
stanchezza per un mondo di cui non apprezzava più i piaceri, né condivideva i dolori. (Flaiano 1988: 666)

En La conversazione continuamente interrotta uno de los personajes principales califica a esta sociedad de posguerra como "una società merdosa che si crede libera, per arrivare a una conclusione En esta situación de crisis, la relación con la mujer, la relación amorosa viene a ser un espejo de la corrupción, de la falta de valores, de la crisis general de la sociedad de la posguerra europe En toda su dramaturgia Flaiano nos presenta una relación amorosa vacía, abocada a la nada. Por eso, no es de extrañar que sus obras estén plagadas de adulterios, de matrimonios de conveniencia, de relaciones amorosa superficiales donde el amor se convierte en sexo, en una manera de seguir la moda o simplemente de aparentar ante la sociedad que uno tiene éxito con los otros. El papel de la mujer como co-protagonista de estas relaciones insulsa no necesita mucha explicación. La conversazione continuanterte interrota es la obra que dedica más espacio las mujeres, tanto en lo que se refiere a personajes vivos dentro de la obra como en cuanto a objeto de reflexión.

En esta última obra publicada por Flaiano, el Poeta nos traza las coordenadas principales que sirven para retratar a la mujer de su tiempo, la que hemos relacionado directamente con el tedio, y para entender las relaciones que los hombre mantienen con ellas:

"Con le donne è inutile nasconderselo, occorre o denaro o tempo. La mia monet è il tempo. Anche tu, se hai tempo, puoi avere tutte le donne che vuoi. Ma devi accompagnarle, parlare, soprattutto ascoltarle. E uscire. Figurati. E poi si mettono delle idee in testa e ti sorvegliano la salute, se fumi, se bevi. (.. Non ci sono problemi, tutte o quasi tutte scopano (...) perché ormai è così l'abitudine, la vita, nell'aria. Pas même avec dignité. Elles couchent, simplement.“ (pp.187-189)

\section{MADRE Y MADRASTRA}

La Amica del Regista (el tercero de los protagonistas de esta obra, junto al Poeta y al Scrittore) es una mujer débil, con grandes deseos de formalizar su relación, con el Regista o con quien sea. No sabemos mucho de ella, sólo que está desesperada: le está llamando continuamente, incluso a la casa del Scrittore donde, teóricamente, están trabajando. Además, sabemos que él no demuestra mayor interés por ella, la evita, no contesta a sus llamadas y, cuando lo hace, es de manera poco cortés, apresurada, de mala gana. Ella sabe que su relación peligra, de ahí su desesperación. En el capítulo quinto su situación es un poco más explícita: se ha quedado embarazada y el Regista quiere que aborte. No es sólo una voluntad, en cierta manera la está obligando a tomar esa decisión. Por otra parte, no sabemos bien cuál es la razón por la que ella no quiere abortar: ¿quiere de verdad un hijo o lo que quiere es que no se ponga fin a una relación que ve casi terminada? Sabe que si aborta ya nada le atará a él. Pero la Amica del Regista no es la única embaraza en esta obra. Y es extraño porque, por regla general, Flaiano no presenta en su obra ni a niños ni a mujeres embarazadas. De hecho, ésta es la única obra en la que aparecen y lo hará en término doble. En La conversazione Flaiano nos presenta a dos embarazadas: la Amica del Regista y Crimilde, la criada del Scrittore. Dos mujeres muy distintas que llevan en su interior una vida nueva, un nuevo ser que podría cambiar la vida de los que lo engendraron. Normalmente las mujeres de Flaiano son todas frívolas, superficiales, preocupadas por mantenerse siempre entre los ricos, famosos, en el mundo de la farándula, siempre en el candelero. Aunque sea a costa de sí mismas.

En general, ellas no son ni artistas ni intelectuales, pero sí se encuentran con artistas o intelectuales. Mujeres jóvenes o de edad media, con dinero, con contactos, sin cualidades de ningún tipo -aunque todas hablen de libros, de cuadros, y lleven dentro de ellas un artista en potencia que nunca aparecerá. Mujeres dedicadas a la 'dolce vita', fiestas y fiestas, recibimientos, encuentros fortuitos. En este panorama los hijos no ocupan un lugar destacado. No tienen tiempo ni ganas. Por eso todas las mujeres que aparecen lo hacen sin niños. Salvo estas dos. Es como si no hubiera lugar para la mujer-madre en esta sociedad que nos presenta Flaiano.

En el caso de la Amica del Regista vemos claramente cómo ese hijo nunca nacerá. Ella es demasiado débil y él demasiado egoista. Terminará convenciéndola para que aborte y luego la abandonará. Ella ya lo sabe pero se intenta engañar. Un hijo nunca les unirá, porque su relación no tiene base, porque no hay amor Sólo soledad por ambas partes y el deseo desesperado de ella de no quedarse sola. El embarazo de Crimilde es otra cosa, porque también esta mujer en nada se parece a las otras mujeres que aparecen en las obras de Ennio. Crimilde es una mujer fuerte, simple. No tiene nada que ver con el mundo de los intelectuales. Es una mujer que trabaja para mantenerse -y en este sentido poco importa que lo haga como asistenta-, una mujer que se entrega a los hombres con alegría, no por rutina. Y que se queda embarazada y tiene el niño. No se plantea en ningún momento -o por lo menos no aparece en la obraabortar. Se ha quedado embarazada y eso es todo. Será madre y lo acepta con alegría como la cosa más natural del mundo. Que no sepa quién es el padre poco importa. Crimilde lleva una vida natural, siguiendo los ritmos naturales de la vida. Y eso es lo que parece atraer al Poeta en este momento de grave crisis personal y profesional: con ella se le abre la posibilidad de llevar una vida normal, al lado de una mujer nada 
complicada que sabe disfrutar de la vida; una mujer con la que seguramente no podrá hablar de literatura, pero que quizás podrá darle la estabilidad y el amor que necesita.

\section{EsPOSA Y AMANTE}

En el teatro de Flaiano no aparece en ningún momento el amor conyugal, parejas casadas sí, prácticamente en cada una de las obras, en todos los textos de amplio respiro, pero nunca existe amor entre ellos. Los personajes son muy explícitos respecto a este tema. El Presidente de La guerra afirma irónicamente que "da noi la guerra sostituisce il divorzio" (p. 100). En Il caso Papaleo, ante la resurrección de su marido, la mujer no muestra sorpresa alguna y ante la premura de Roberto para que le saque de la tumba lo antes posible, ella llegará a preguntarle: "Posso venire subito o vuoi che venga domattina? Dicevo che ho l'influenza, ma vengo subito" (p. 155).

El matrimonio como un doble nudo en la vida: cada uno está enamorado de una persona soñada, inexistente, pero que no es precisamente su cónyuge. No se conoce a la persona que se tiene al lado: al contrario, se la desprecia, se la olvida y se crea un personaje ficticio. De ahí que el Scrittore de La conversazione continuamente interrotta afirme: "Ci si sposa in quattro" (p. 182): dos personajes verdaderos y dos imaginarios. Pero si hemos asistido a la triste realidad del matrimonio, el adulterio tampoco dará sentido a las vidas de los personajes de Flaiano. Los amantes de Un marziano a Roma, La donna nell'armadio y La conversazione continuamente interrotta conducen vidas frívolas y poco satisfactorias. Tan sólo, en algún momento, algún personaje intenta realmente amar, establecer con la mujer una relación satisfactoria. Es Roberto en Il caso Papaleo y Adriano y Anna en Un marziano a Roma. En esta última obra, un Adriano enamorado le canta a Anna:

"Andiamo, donna, è tempo d'amare,

sul raccordo anulare.

La campagna ci attende, il cacciatore

E il fido pastore.

Andiamo, donna, togliti il rossetto.

Offriti. Aspetto.

Cosi furtivo, il desiderio passa,

Nell'uomo-massa."(p.70)

Al final, como vemos ya en la canción, todo quedará en un furtivo intento sin consecuencias.

\section{LA MUJER Y LA MARAVILLA}

Junto a este concepto de mujer estrechamente relacionado con el aburrimiento y el tedio, nos encontramos ante el concepto de mujer como maravilla, simplicidad, alegría, autenticidad. Este sentimiento de maravilla ante la mujer y el amor de que solamente ella es capaz tiene dos caras: por un lado, la atracción por lo instintivo, por lo natural, por lo primitivo, por la belleza; por el otro, el miedo ante lo desconocido, ante lo auténtico -de lo que se encuentra uno tan alejado- e incluso la presencia de la enfermedad y de la muerte. En Un marziano a Roma Fabrizio afirma:

"La donna mi attira e mi spaventa sempre, come una jungla dalla quale non sai se ritorni vivo. Sabbie mobili, pantere, fiori stupendi e mortali. Quando ti amano è peggio. Nel loro amore c'e la minaccia celata, il veleno che agirà dopo. Vogliono ucciderti, questo è il loro scopo. (...) Nell'amore c'è qualcosa di umido, di viscerale, diconturbante Tisenti preso, offeso, umiliato, aneli alla pace e vuoi che il suppliz continui, tremi che possa finire, all'idea del vuoto che seguirà" (p.80)

Se trata de una referencia explícita a Tempo di uccidere y a la relación del oficial italiano con una mujer etíope en el bosque: una mujer capaz de un amor puro, que no espera nada a cambio y que lo da todo; es el amor absoluto, total que, para Flaiano, es lo único que salva, que da sentido a una vida en la que han desaparecido los valores, los sentimientos, la moral; ${ }^{2}$ pero también, una mujer portadora de enfermedad, de veneno, de mal, de muerte ${ }^{3}$. De esta manera, la obra dramática de Flaiano enlaza con su obra narrativa y, de manera especial, con Mariam, la etíope de Tempo di uccidere, la mujer que provoca en el protagonista este sentimiento de admiración, de maravilla ante lo instintivo, ante esos dos mil años atrás donde todavía las cosas eran auténticas. Es la maravilla y el deseo de lo primitivo, de lo aútentico lo que atrae al personaje principal de la novela irremediablemente a esa mujer. Porque ella es la única que le podrá enseñar el valor de tantas cosas, la otra mitad de la verdad que busca desesperadamente Antonio en La donna nell'armadio. En Tempo di uccidere el oficial italiano protagonista de la novela ha encontrado esa verdad que buscaba y que ha dado otro sentido a su vida, porque ha encontrado lo

Flaiano considera que en el amor está la solución a esta crisis personal y social que atravesamos. Pero se trat de un amor concreto: el amor absoluto, total. Así lo afirma el autor en una entrevista concedida a Giulio Villa Santa: “La via (di scampo) non può essere che l'amore, ma non l'amore canino, cinico: l'amore assoluto, totale. L'amore che comincia da sé e va verso gli altri, che comprende i giorni, che comprende il tempo che abbiamo vissuto, comprende gli amici che ci hanno abbandonato, che sono morti, comprende le persone che abbiamo conosciuto, comprende anche le persone che non conosciamo. (...) Non vedo altra via di scampo perché tutte le altre suggerite dalle contingenze, suggerite dal nostro tono di vita ormai degradato, non danno un'altra indicazione." (Flaiano 1988: 1236-1237)

3 Ha sido precisamente este aspecto más negativo del amor el que ha llevado a creer en una visión pesimista del amor. Veamos la entrevista mantenida con Italo Alighiero Chiusano donde éste pregunta a Flaiano por su visión pesimista del amor: "CHIUSANO: Nei suoi libri c'è sempre una visione pessimistica dell'amore. Si tratta di uomini soli che hanno un breve incontro erotico dalla lebbra o dalla metamorfosi canina o dal disgusto o che addirittura vengono rapiti nello spazio e non vedono l'ora di tornare a terra. Perché? FLAIANO: Perché, vede, l'amore è solo un momento, un momento fugacissimo. Appena passato, si trasforma, diventa mostruoso, non ci lascia nemmeno un po' di gratitudine. Altra cosa è il matrimonio. E raro che riesca, ma quando riesce è la cosa più bella che esista. Però sembra che gli uomini ormai non lo vogliano più. (Flaiano 1988:1221)

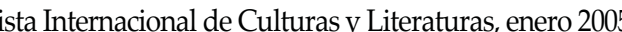


auténtico, la mujer y el amor sin complicaciones, instintivo, espontáneo, que sigue los cánones de la naturaleza y no las convenciones de la sociedad.

El protagonista se ha puesto frente a ese amor, que es belleza y que es el bien, pero en el que también está presente la enfermedad y la muerte. El oficial terminará por aceptar ese amor, quizás porque estaba dispuesto a aceptarlo ya desde un principio, ya que, a pesar de ser muerte, ese amor auténtico conduce a la salvación del hombre, es la única salida a esa vida de continua crisis en todos los ámbitos. Pero no es una salida fácil. No lo será para Adriano, el personaje de Un marziano a Roma más dispuesto al cambio y a la renovación, con respecto a Anna, ni para los protagonistas de La conversazione continuamente interrotta, incapaces de renunciar a su situación para llegar a la autenticidad. El intento de Roberto en Il caso Papaleo de volver a empezar su vida con Angela no dará tampoco sus frutos. También el Scrittore en La conversazione continuamente interrotta traza una imagen de lo que es la mujer para él. Evidentemente, se trata de una mujer que ve en un sueño:

"Quella donna del sogno. (...) Quella donna mi turba ancora. Era stupenda, meravigliosa, ho ancora nelle orecchie il suo riso chiaro, dolcissimo, invitante." (p.183)

Este ideal de mujer aparece en otras obras de Flaiano y muy especialmente en Il caso Papaleo cuando Roberto habla de Angela. Un amor verdadero que sólo aparece en momentos no reales, imaginarios, soñados, o ante la realidad de la muerte, como en Il caso Papaleo: Roberto se despierta de la muerte con el recuerdo de un amor juvenil, lo único que realmente da un sentido a su vida y por lo que ha merecido vivir. Porque con relación a las otras historias de 'amor' que ha tenido -incluido su matrimonio- esa mujer clave de su vida "era la verità, l'acqua fresca quando si ha sete" (p. 157). Este es el ideal de mujer y de un amor sin clichés, auténtico, espontáneo, que no sigue los cánones de la sociedad. Es la búsqueda constante de lo auténtico, de una mujer que sea la verdad, pura como el agua fresca, que no traicione, que sea fiel, siempre disponible.

Ese era el amor que recordaba Roberto Papaleo y que ahora, tras la experiencia de la muerte, es el único punto de referencia importante de toda su vida. Pero aún entonces, ese amor tan sólo será válido para un componente de la pareja. El otro se mantendrá al margen, ausente total de ese amor, sin memoria. Es más, Angela Rocco siente lo mismo que ha expresado Roberto, pero por otra persona. Las palabras que utilizará Angela para definir ese amor maravilloso serán prácticamente las mismas que utilizó Roberto:

"Era la gioia di vivere, nessun uomo mi ha dato tanto, lo amavo, anzi lo ammiravo, mi riempiva di meraviglia per la serenità che rendeva tutto semplice" (p. 160).
LA MUJER-PERRO

En estrecha relación con el concepto de amor como maravilla, en estas obras se hace referencia al ideal de mujer-perro ${ }^{4}$ que aparece de manera más desarrollada y precisa en la narrativa de Flaiano: en Tempo di uccidere y Melampus. Sobre ello, Sergiacomo afirma:

"La relazione cane-donna è un procedimento analogico simbolico caro a Flaiano ed è funzionale a comprendere il suo ideale femminile da contrapporre alla sagra della femminilità in negativo (...). Il cane compare con frequenza nelle pagine di Flaiano con un significato ambivalente di ferocia animalesca ma anche di tenerezza e fedeltà (...). L'amore inteso come cieca, animalesca sottomissione si esprime nella metafora canina e trova la sua incarnazione nella bestiale dedizione della donna al suo uomo". (Sergiacomo 1993:117-118)

Esas características: ternura, fidelidad, sumisión y absoluta entrega al hombre, a la vez que ferocidad animal y ciega, atribuidas a Mariam en Tempo di uccidere y, sobre todo, a Liza en Melampus, aparecen claramente en Il caso Papaleo. Roberto se confiesa ante Angela:

"Non ho mai potuto dimenticarti, proprio per questa rara qualità, che tu sola possiedi, di non fare mai le cose difficili (...). Quelle che tu mi suggerisci sono mmagini pulite, oneste (...). Non ho amato che te... Anzi, è piu dell'amore. La meraviglia, l'ammirazione per il fatto che esistevi" (pp. 158-159).

Esta mujer-perro aparece también en Un marziano a Roma: la sumisión alegre y total que encontramos en Patrizia y Mara ante el marciano. Para Flaiano, ante la relatividad del amor, la entrega absoluta y 'canina' es la única salvación.

"MARA: Lo amo sì, ma se togli gli attimi fuggenti, che ci resta?

PATRIZIA: Ci resta tutto: servirlo, volergli bene, classificare la corrispondenza. MARA: La nostra vita disordinata aveva il suo fascino PATRIZIA: Non la rimpiangere. Qui siamo su un altro piano, qui c’è il fascino dell'assoluta dedizione" (pp. 33-34)
Se trata del ideal de mujer y de un amor de verdad, espontáneo, que no sigue los
cánones de la sociedad. Es la búsqueda constante de lo auténtico, de una mujer que

Se trata del ideal de mujer y de un amor de verdad, espontáneo, que no sigue los
cánones de la sociedad. Es la búsqueda constante de lo auténtico, de una mujer que

\begin{abstract}
4 En la entrevista concedida a Villa Santa, Flaiano explica qué es lo que entiende por mujer-perro: La donna che si trasforma in cane da lei ricordata è una speranza che per salvare qualche cosa bisogna cominciare a rinunciare a quello che noi siamo, cioè alla nostra 'cultura' tra virgolette: perché la vera cultura è un'altra, è quello che sanno gli analfabeti in un certo senso, cioè come vivere nell'ambiente, come con offenderlo, come rispettare gli altri. Questa è cultura; e parlando di amore, non vorrei che lei equivocasse. L'amore qui è inteso come un fatto estremamente ampio, estremamente divino; è l'amore che comprende oprattutto la possibilità che noi un giorno non possiamo più esercitarlo, perché noi un giorno ce ne
ndremo e dobbiamo lasciare di noi un ricordo che sia perlomeno decente." (Flaiano 1988: 1236-1237)
\end{abstract}


sea la verdad, que no traicione, que sea fiel, siempre disponible -en un momento en que el adulterio era casi una obligación y que aparece constantemente en todas las obras de Flaiano: el adulterio como un ejemplo más del tedio, del sinsentido de la vida. Esta mujer portadora de amor absoluto es la única esperanza para el hombre, porque, como afirma el autor en La solitudine del satiro, la única posibilidad de salvación se encuentra en el amor absoluto, total.

La Moglie del Scrittore puede ilustrar una teoría de Flaiano sobre la mujer-perro, la mujer dedicada exclusivamente al hombre en cuerpo y alma. Una mujer que ve a hombre, por un lado, como su dueño y señor y a cuya voluntad se somete y, por otro como un ser que necesita protección: exactamente como un perro mastín a su dueño En el caso de la Moglie del Scrittore su sueño de mujer-perro se presenta precisamente como un sueño de la infancia y de la adolescencia. A la pregunta de por qué se ha casado con su marido ella responderá:

"Non so. Per una serie di equivoci. Una volta mi dette uno schiaffo, me ne innamorai. Speravo che mi picchiasse. Tutte le ragazze ai miei tempi sognavamo un vero uomo (...), un uomo da salvare, da proteggere. Uno di quelli che la sera vanno a bere con gli amici e la moglie deve andare a ritirarli, verso l'una, ubriachi." (p.176)

Seguramente querría y quiere un hombre que no tenga nada que ver con esos intelectuales ineptos que la rodean.

Io sono felice. Ma pensavo a un uomo che la sera tornasse stanco, sporco, e che si lavasse in cucina. (...) E che trasalisse ad ogni suono di campanello. Un uomo con certo passato." (p.177)

La 'mujer-perro'también aparece en La conversazione continuamente interrotta en la figura de la Amica del Regista, evidentemente no de manera tan fulminante como en Melampus, pero el personaje comparte algunos rasgos de ésta. Una dedicación absoluta al hombre: le quita los zapatos (p.203), le pone un cojín debajo de la cabeza para que esté cómodo (p.204) e incluso después de la terrible discusión que tiene acerca de abortar o no, ella está dispuesta a hacerle algo de comer.

Erílogo: la Ofelia de Flaiano

Para terminar y a modo de conclusión me gustaría hacer una breve referencia a la mujer en el mito de Hamlet y cómo éste aparece reinterpretado en la dramaturgia de Flaiano. La Ofelia de Hamlet aparece en dos de sus textos teatrales : Amleto ' 43 y La conversazione continuamente interrotta.
La primera obra es muy breve. Se trata de la interpretación personal que hizo Flaiano de la historia de Hamlet, quizá como habría sido en una época como la de la posguerra italiana $^{5}$, sin valores ni referentes absolutos, donde todo es relativo, incluso el amor, o, quizá, es la propia tragedia de Shakespeare desnuda de toda mistificación. Ésta es, entonces, la versión de la nueva Ofelia, la que viviría en esta época en la que escribe Flaiano, y cómo la vería un Hamlet de ahora:

"Quanto a te, Ofelia, ti detesto.

Vorresti fare di me un principe ereditario, un taglianastri, un giramondo, un

rubacuori!

Tu ignori ch'io sono un Prometeo

Senza fegato e senza avvoltoio.

(Ofelia impazzisce)

Come? Di già impazzita?

Ofelia! Tu anneghi in un bicchier d'acqua!" (p. 258).

Se trata, como vemos, de una Ofelia histérica, que quiere hacer de Hamlet algo que no es y que no quiere ser. Una Ofelia de la que Hamlet no está enamorado y que no representa el prototipo de mujer auténtica, pura, capaz de un amor de verdad. La Ofelia de nuestra época sería más bien, la que recuerda el Poeta en La conversazione continuamente interrotta:

"La prima volta che vidi L'Amleto, in un teatro ambulante, avevo otto o nove anni, nella parte di Ofelia la figlia del capocomico, una ragazza incinta. Incinta di sette, otto mesi. Me ne innamorai di colpo perdutamente. Ho continuato a credere che questa fosse la realtà di Ofelia" (p. 167)

En esta obra de Flaiano, Ofelia -sin Hamlet- es la asistenta del Scrittore. Se llama Crimilde, pero desde el primer momento el Poeta la llama Ofelia porque también ella -como la actriz que interpretaba Ofelia- está en avanzado estado de gestación. Una joven que "non ha saputo mai dire di no a nessuno" (p. 170). En el futuro de esta joven, el Poeta ve la continuación de la verdad de Hamlet y Ofelia. Por eso exclama:

"Una donna così andrebbe sposata immediatamente. Ha bisogno di sicurezza, di stabilità. Qui possiamo parlare di innocenza, questo sì che è un fatto d'amore, pieno, assoluto. Se non vivessi solo, la sposerei io. Se non altro per riparare i torti di quel buffone, di quel refoulé di Amleto!" (p. 170). Y, efectivamente, se casará (n) proprie grazie! Che c'è di meglio? Questo è amore" (p. 190).

\section{REFERENCIAS BIBLIOGRÁFICAS}

Antonucci, G., "Flaiano e il teatro”, en Sergiacomo, pp. 131-140., 1993.

5 De hecho, antes de empezar la obra Flaiano escribe: "Nell'ottobre del '43 non si parlava di teatro della crudeltà, ma i tempi erano abbastanza crudeli. Scrissi allora questo piccolo Hamlet”. (p.258)

作 
Atti del Convegno nazionale nel decennale della morte dello scrittore Ennio Flaiano, L'uomo e l'opera, Pescara, Ediars, 1982.

Atti del Convegno, Flaiano e il tempo del “Mondo”, Pescara, Ediars, 1989.

Atti del Convegno nazionale, Tempo di uccidere, Pescara, Ediars, 1994.

Atti del Convegno nazionale, Il teatro di Flaiano, Pescara, Ediars, 1995.

Ciarletta, N., "Il teatro di Flaiano", en Atti, pp. 93-98., 1982.

Feo de, S., "Un marziano a Roma”, en Sergiacomo, pp. 252-253, 1992.

Flaiano, E., Opere: scritti postumi e scritti 1947-72, volumi I e II, Milano, Classici Bompiani, 1988.

----, Un marziano a Roma e altre farse, Torino, Einaudi,1971.

Geron, G., "La conversazione continuamente interrotta”, en Atti, pp. 29-33, 1995.

Laurenzi, C., "Tutto il teatro di Flaiano”, Il Dramma, aprile 1972.

Mango, A., "Conversazione continuamente interrotta”, Il Sipario, agosto-settempre 1972.

Moretti, V. (ed.), Flaiano e “Oggi e Domani”, Pescara, Ediars, 1993.

Pensa, C. M., "Un marziano a Roma”, en Atti, pp. 20-23, 1995.

Poesio, P. E., "Flaiano e le sue farse”, en Atti, pp. 34-40, 1995.

Pullini, G., "La critica al teatro di Ennio Flaiano (1946-1993), en Atti, pp. 55-68, 1995.

Rebora, R., "Vocazione mancata di un marziano", Il Sipario, gennaio, 1961.

Salce, L., "La conversazione continuamente interrotta”, en Atti, pp. 99-104, 1982.

Sergiacomo, L., (ed.), Flaiano vent'anni dopo, Pescara, Ediars, 1993.

----, “Le donne raccontate da Flaiano”, en Sergiacomo, pp. 109-120., 1993.

----, (ed.), La critica e Flaiano, Pescara, Ediars, 1992.

Tian, R., "Il teatro di Flaiano", en Atti, pp. 7-12, 1995. 